\title{
AMMONOIDEOS DEL DEVÓNICO INFERIOR Y MEDIO EN EL PIRINEO ORIENTAL Y CENTRAL. ANTECEDENTES HISTÓRICOS Y NUEVOS HALLAZGOS
}

\author{
J. Román MONTESINOS y Javier SANZ LÓPEZ \\ Área de Paleontología. Facultad de CC. de la Educación, Universidad de A Coruña. Paseo de \\ Ronda 47, 15011 A Coruña. Correo electrónico: roman@udc.es / jasanz@udc.es
}

\begin{abstract}
Montesinos, J.R. y Sanz López, J. 1999. Ammonoideos del Devónico Inferior y Medio en el Pirineo oriental y central. Antecedentes históricos y nuevos hallazgos. [Lower and Middle Devonian ammonoids from the centraleastern Pyrenees. Historical precedents and new findings]. Revista Española de Paleontología, $\mathbf{n}^{\circ}$ extr. Homenaje al Prof. J. Truyols, 97-108. ISSN 0213-6937.
\end{abstract}

\begin{abstract}
The ammonoid taxa Agoniatites, Anarcestes, Latanarcestes, Erbenoceras, Mimagoniatites, Pinacites and Sellanarcestes from the Lower and Middle Devonian of the Pyrenees are described and illustrated. Some of these taxa (Erbenoceras, Sellanarcestes and Pinacites) are new findings for this area. An historic review of references about Emsian-Eifelian ammonoids from the Pyrenees is given and the stratigraphic position and significance of the "Anarcestes horizon" of Dalloni (1911 et seq.) is discussed. The biostratigraphic position of the fauna is specified in the stratigraphic context and its relation with conodont associations is described. The lithostratigraphic units used in this work are described and defined.
\end{abstract}

Keywords: Ammonoids, biostratigraphy, lithostratigraphy, Lower Devonian, Middle Devonian, Pyrenees.

\section{RESUMEN}

Se describen y se figuran varios taxones de ammonoideos (Agoniatites, Anarcestes, Erbenoceras, Latanarcestes, Mimagoniatites, Pinacites y Sellanarcestes) procedentes de series estratigráficas del Devónico Inferior y Medio del Pirineo central y oriental. Algunos de ellos, Erbenoceras, Sellanarcestes y Pinacites, son la primera cita en el ámbito geográfico estudiado. Se realiza una revisión histórica sobre las escasas citas de ammonoideos de autores previos y se discute el significado y alcance del "horizonte de Anarcestes" de Dalloni (1911 et seq.). Se precisa además la posición de la fauna estudiada en un contexto estratigráfico y se relaciona con las asociaciones de conodontos obtenidas en las mismas secciones. Para ello y en algunos casos, ha sido necesaria la definición y descripción de las diferentes unidades litoestratigráficas utilizadas en el texto.

Palabras clave: Ammonoideos, bioestratigrafía, litoestratigrafía, Devónico Inferior, Devónico Medio, Pirineos.

\section{INTRODUCCIÓN: ANTECEDENTES Y SIGNIFICADO}

La información existente sobre ammonoideos devónicos en el Pirineo es escasa. Las citas son en extremo puntuales, debido quizás tanto al deficiente estado de conservación de la macrofauna como a la carencia de un muestreo sistemático de los distintos afloramientos devónicos pirenaicos. Por otro lado, una gran parte de las referencias bibliográficas son antiguas, sin descripciones e ilustraciones y no indican de forma precisa la situación estratigráfica de las faunas.

Dalloni (1910 y 1911) fue el primer autor que citó en sus trabajos de forma generalizada la presencia de ammonoideos del Devónico Inferior-Medio. Una parte de los ejemplares estudiados por este autor proceden de capas carbonatadas de los Pirineos aragoneses, posiblemente de la parte alta de la Formación Formigal de Valero (1974). En particular, Dalloni (1910) identificó Agoniatites sp. en las calizas nodulares y margas del Pasino y Anarcestes subnautilinus Beyrich [=Latanarcestes noeggerathi (Buch)] y Agoniatites sp. en las calizas nodulares rojas y verdes de Tramacastilla, asignando el conjunto de fauna al Eifeliense. Con posterioridad, y en otras localidades de la misma región (Escarra y Tarmañones), Wensink (1962) delimitó un 


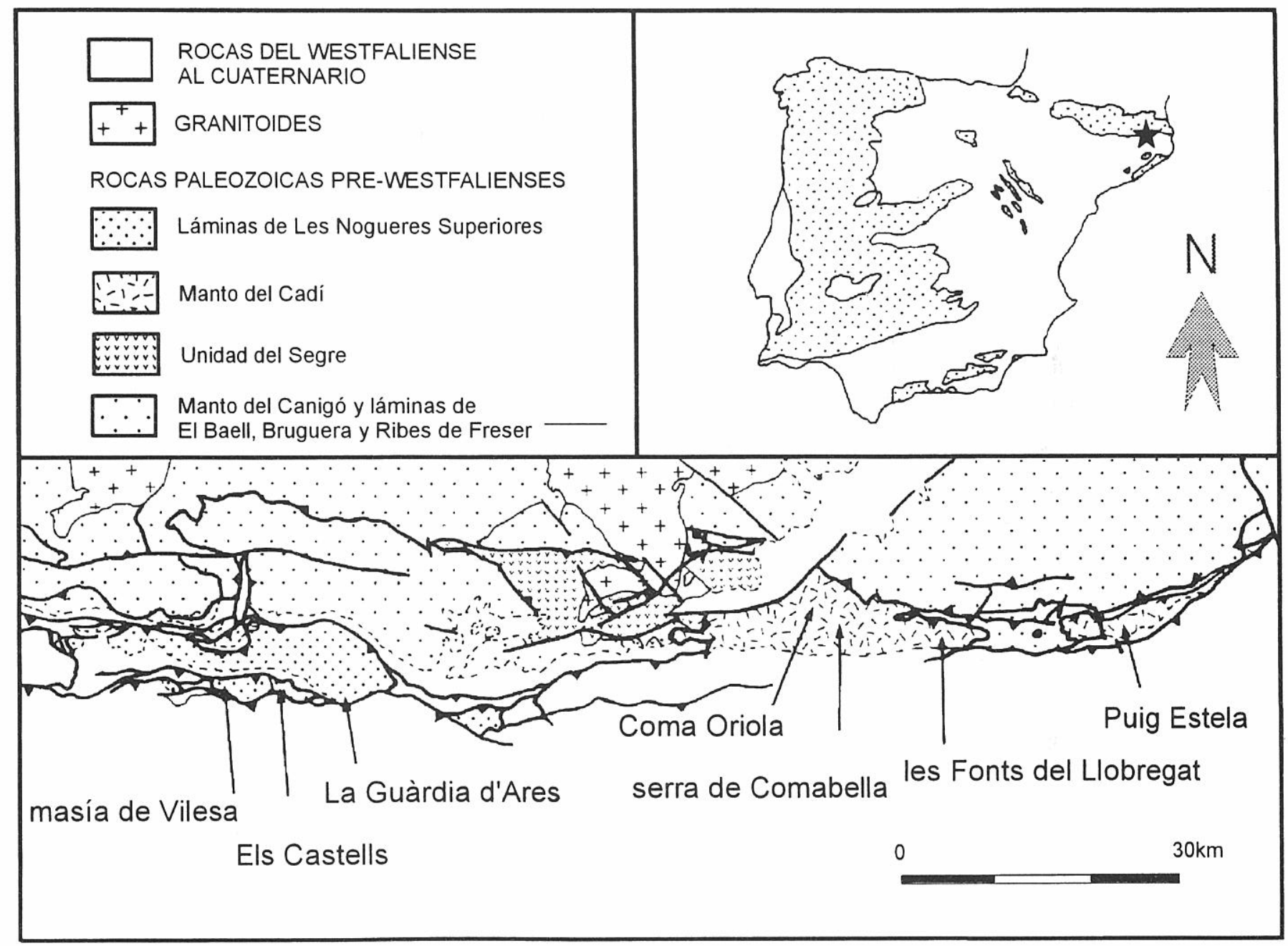

Figura 1. Posición del área estudiada en la península Ibérica y esquema geológico del Pirineo central y oriental meridional con la ubicación de las unidades tectónicas utilizadas en el texto y donde se han señalado las diferentes localidades de las que proceden los ammonoideos estudiados y las secciones estratigráficas de la figura 2.

tramo menor que 50 metros de calizas de tonos grises a grises anaranjados entre lutitas y margas, donde determinó Goniatites subnautilinus (=Latanarcestes noeggerathi), Agoniatites sp. y Bactrites cf. carinatus Sandberger. El mismo autor también determinó Agoniatites sp. en una alternancia de calizas rojas y moradas con margas, alternancia que situó bajo la Caliza de Corales al sur de la Peña Foradada. Wensink (1962) atribuyó ambos tramos al Cuviniense inferior.

En sucesivos trabajos, Dalloni (1911, 1913 y 1930) citó asociaciones de ammonoideos y otros cefalópodos en el Pirineo central meridional: Anarcestes subnautilus, A. lateseptatus (Beyrich), A. lateseptatus var. plebeia (Barrande), Agoniatites sp., Jovellania sp., Phragmoceras subventricosum de Verneuil y d'Archiac y Bactrites sp. Estas citas pertenecen a localidades situadas en diferentes láminas de Les Nogueres Superiores (Fig. 1): unidad de Els Castells (cercanías de Useu y Buseu) y unidad de El Comte-Freixe (proximidades de Castellàs; entre La Guàrdia d'Ares y Noves; cerca del Coll del Cantó, Trejuvell; Roc dels Pixutets y bajo el Pico de Bellpui). En el Pirineo oriental, Dalloni (1930) determinó Anarcestes sp. y Bactrites sp. entre Torres y Ansovell, en la unidad tectónica del Segre.

La gran extensión geográfica de las capas con ammonoideos condujo a Dalloni (1911) a utilizar el término de "horizonte con Anarcestes subnautilinus". Este tramo u horizonte correspondía, según el mencionado autor, a un cuerpo de calizas nodulares típicas que se encontraban sobre la base del Eifeliense, contenía elementos abundantes de Anarcestes y Agoniatites, y tenía una notable extensión geográfica a lo largo de diferentes afloramientos de la vertiente meridional pirenaica. Según el mismo autor, estas calizas con ammonoideos presentaban un cambio lateral de facies a las calizas rojas con Paraspirifer cultrijugatus (Roemer) en el Pirineo aragonés.

Las referencias sobre ammnoideos del Emsiense Inferior del Pirineo son aún más escasas. Schmidt (1931) identificó Mimagoniatites ? falcistria Fuchs en las lutitas negras de Hount de Ver o lutitas de Cathervielle (Pirineo central francés). Sin embargo, para Bouquet y Stoppel (1975) estas lutitas deben ser asignadas al Eifeliense, ya que contienen Goniatites ex gr. Anarcestides (determinación de Erben y Haas dónde, posiblemente, Anarcestides haga referencia a Anarcestidae). 
Finalmente, Kullmann y Calzada (1982) describieron un tramo de calizas con lutitas grises cerca del Taga (Manto del Cadí) que puede ser equivalente a la parte inferior de la Fm. Villech. Estos autores identificaron en una muestra suelta de este tramo y en la pista de Coll de Jou, los cefalópodos Mimosphinctes cf. cantabricus Kullmann y Mimagoniatites sp., que asignaron al Emsiense Inferior alto.

Recientemente, se han recolectado ejemplares de ammonoideos del Devónico Inferior y Medio en diferentes secciones del Pirineo oriental y central (Fig. 1). La revisión de estas formas, junto a su situación estratigráfica, presenta un innegable interés. Más aún, cuando estos hallazgos se encuentran próximos a asociaciones de conodontos.

\section{MARCO GEOLÓGICO}

La mayoría de los hallazgos de ammonoideos proceden de capas de las formaciones Villech y Comabella (Fig. 2).

La Formación Villech (Hartelvelt, 1970; ver anexo) se encuentra sobre las calizas grises de la Fm. Castanesa (Mey, 1967) y por debajo de la Fm. Comabella (ver anexo). La potencia de la Fm. Villech varía entre 30 y 75 $\mathrm{m}$, y está integrada por alternancias decimétricas a centimétricas de margas rojas y verdes y calizas versicolores. La fauna está dominada por dacrioconáridos, aunque se encuentran también fragmentos de trilobites, crinoides, briozoos, ostrácodos, gasterópodos, algunos ortocerátidos y escasos corales.

La Formación Calizas de Comabella (ver anexo) está compuesta por calizas nodulares versicolores, entre las que dominan las calizas de tonos grises. En algunas secciones de la Sierra de Moixeró, en la parte media y superior de la formación, predominan capas de calizas crinoidales y calizas con corales.

Las formaciones Villech y Comabella se extienden entre Camprodon y la Noguera Pallaresa. Según Mey (1968), Boersma (1973) y Zwart (1979) en esta región, las sucesiones devónicas y carboníferas son similares y las agruparon en la denominación de "Área de subfacies de El Comte". Desde el punto de vista estructural, la región aludida corresponde a: 1) la unidad tectónica del Segre de Casas et al. (1989), 2) un conjunto de láminas cabalgantes suprayacentes, compuestas por el manto alpino del Cadí (Muñoz, 1985) y 3) las escamas de El Comte-Freixe, Arcalís, Baro y Erdo (Seguret, 1972), que forman parte de las láminas alpinas de Les Nogueres Superiores de Muñoz (1992). En cambio, existen otras escamas de Les Nogueres Superiores, como la de Els Castells, con sucesiones devónicas diferentes, que Boersma (1973) incluyó en el "Área de subfacies de Sierra Negra".

La sucesión estratigráfica en la sección de Els Castells (unidad tectónica de Els Castells, Figs. 1 y 2) contiene una fauna abundante de conodontos, dacrioconáridos y trilobites. Sobre la Formación Castanesa se disponen 30 metros de lutitas, margas y calizas nodulares ocres en capas centimétricas a decimétricas. Estas alternancias corresponden a las Capas de Els Castells (ver anexo). La sucesión de alternancias de lutitas y carbonatos rojizos suprayacentes a las capas de Els Castells, con una potencia de 50-60 m, se incluye aquí en la Formación Villech.

Por encima de la Formación Villech, se dispone un tramo de calizas nodulares y calizas de coloraciones claras (17 metros) pertenecientes a la parte inferior de la Fm. Comabella. Sobre el tramo anterior, yace una alternancia de lutitas y margas con algún nivel calcáreo, que constituye las Capas de Taús (ver anexo). Estas capas representan un cambio lateral de facies de la parte media de la Fm. Comabella, con respecto a la tónica general del las sucesiones del "Área de subfacies de El Comte".

En la parte alta de las Capas de Taús, existen algunos niveles con nódulos que contienen una asociación de conodontos (Polygnathus costatus costatus Klapper, $P$. costatus $\rightarrow P$. pseudofoliatus Wittekindt, $P$. linguiformis linguiformis Hinde e Icriodus regularicrescens Bultynck) de la Zona de Tortodus kockelianus australis de Klapper (1977), Eifeliense. Sobre este tramo se dispone un paquete de calizas nodulares de dacrioconáridos con características propias de la parte alta de la Fm. Comabella y cuya base ha proporcionado conodontos (Polygnathus pseudofoliatus Wittekindt, $P$. pseudofoliatus $\rightarrow P$. ensensis Ziegler y Klapper, $P$. $l$. linguiformis Hinde e Icriodus arkonensis Stauffer) de la Zona de Polygnathus ensensis de Weddige (1977).

\section{LOCALIZACIÓN Y EDAD DE LA FAUNA ESTUDIADA}

\section{I) MANTO DEL CADÍ}

\section{Sección del Puig Estela}

En la sección del Puig Estela (Figs. 1, 2), al norte de Ogassa, ha sido hallado un ejemplar de Erbenoceras ex gr. filalense (G. Termier y H. Termier) en la parte inferior de la Fm. Villech, dentro de las alternancias basales de calizas y margas rojas. El ejemplar procede de una capa que se ubica 3 metros por encima de la muestra de conodontos O19, asignada por Sanz (1996) a la Subzona Superior de Polygnathus gronbergi (Bultynck, 1989), y 6 metros bajo la muestra $\mathrm{O} 22$, correlacionada por el mismo autor con la Zona de P. inversus de Bultynck (1989). Estos datos estarían en posible concordancia con la posición estratigráfica descrita para el conjunto de la "Fauna de Anetoceras" de Chlupác (1976) y Chlupác y Turek (1983), que engloba a los ammonoideos más primitivos, y que correlacionan con la Biozona de $P$. gronbergi de conodontos. En el marco de la Península Ibérica, estas capas serían comparables con las alternancias superpuestas al Miembro Requejada de la Formación Abadía, en el Dominio Palentino (Cordillera Cantábrica) en las que se encuentran Erbenoceras filalense $\mathrm{y}$, en su parte más alta, Mimosphinctes cantabricus Kullmann. 
Esta es la segunda vez que se citan elementos de la "Fauna de Anetoceras" en capas del Devónico del Pirineo y dentro de la misma unidad tectónica: el manto del Cadí. La primera cita corresponde a Kullmann y Calzada (1982) (ver introducción) quienes determinaron Mimosphinctes cf. cantabricus y Mimagoniatites sp. De todas formas, la presencia de Erbenoceras Bogoslovski es primera cita en el ámbito geológico del Pirineo.

En la parte media alta de la Fm. Villech, se ha determinado Mimagoniatites sp. A. Este ejemplar fue obtenido $5 \mathrm{~m}$ por encima de la muestra O26 con Polygnathus linguiformis bultyncki Weddige, muestra de conodontos correlacionada con un intervalo dentro de la parte inferior de la Zona de Polygnathus serotinus de Klapper et al. (1978); y diez metros por debajo de capas con elementos de la parte superior de la Biozona de $P$. serotinus. La edad atribuida por tanto al ejemplar es Emsiense Superior.

Los últimos 6 metros de la Fm. Villech en el Puig Estela han proporcionado varios especímenes. Un ejemplar de Sellanarcestes sp. se encuentra $1 \mathrm{~m}$ por debajo de la muestra de conodontos $\mathrm{O} 34$, asignada a la parte superior de la Zona de $P$. serotinus. Inmediatamente por encima de $\mathrm{O} 34$ se ha determinado Latanarcestes sp., y entre 2 y 2,5 metros se han encontrado dos ejemplares de Anarcestes sp. Los hallazgos se encuentran intercalados con muestras de conodontos correlacionadas con la parte superior de la Zona de $P$. serotinus de Klapper et al. (1978).

\section{Secciones en el área de Castellar de N'Hug}

Los tramos culminantes de la Fm. Villech y los primeros metros de la Fm. Comabella en Les Fonts del Llobregat, L'Erola de Castellar, Coll de Les Tortes y el Rieral suministraron varios ejemplares en muy mal estado de conservación. Tan sólo en la parte inferior de la Fm. Comabella, en el torrente del Rieral, se ha determinado Agoniatites (Paraphyllites ?) sp. en tramos que fueron correlacionados con la Zona de Polygnathus costatus patulus de Weddige (1977), Emsiense Superior.

\section{Sección de Serra de Comabella}

En la sección de Serra de Comabella, entre la Tosa d'Alp y el Puigllançada, se han observado varios horizontes con ammonoideos. Un ejemplar de Mimagoniatites ? sp. se ha obtenido 16 metros bajo el techo de la Fm. Villech, justo por encima de la muestra de conodontos $\mathrm{C} 45$, atribuida a la Zona de P. serotinus o a la base de la Zona de P. c. patulus por Sanz (1996). Por encima, en un tramo entre 5-10 metros bajo el techo de la Fm. Villech, se han determinado Anarcestes ex gr. lateseptatus y Anarcestes sp., correlacionado con el mismo intervalo de conodontos.

En la parte inferior de la Fm. Comabella, existen capas centimétricas de margas rojizas intercaladas entre calizas nodulares que han suministrado un ejemplar de Pinacites cf. eminens Chlupác y Turek. El espécimen procede de un nivel atribuido a la Zona de Polygnathus costatus costatus de Weddige (1977) en el Eifeliense, ya que se encuentra unos cuatro metros sobre la muestra C68 con Polygnathus costatus costatus y P. c. patulus Klapper, asignada a la Zona de $P$. c. costatus y 5 metros bajo la muestra C70 que contiene $P$. costatus costatus, $P$. c. patulus transicional a $P$. pseudofoliatus, $P$. linguiformis linguiformis y un ejemplar asignado con dudas a Tortodus intermedius (Bultynck), asociación correlacionada por Sanz (1996) con un intervalo en la parte superior de la Zona de $P$. c. costatus o ya con la Zona de T. $k$. australis. Esta posición bioestratigráfica es acorde con la que ocupa el taxón nominal en Bohemia, en capas de la Caliza de Chotec (cf. Chlupác y Turek 1977 y 1983).

\section{Sección de la Coma Oriola}

La Fm. Villech tiene un carácter más carbonatado y una menor potencia en otras secciones cercanas a la Tosa d'Alp, como en la sección de la Coma Oriola. En esta sección tan sólo se ha detectado un horizonte con abundantes cefalópodos, localizado tres metros bajo el techo de la formación. Contiene Anarcestes ex gr. lateseptatus asociado a conodontos de un intervalo indeterminado entre la Zona de $P$. serotinus y la parte basal de la Zona de P. c. patulus. Se han encontrado ejemplares típicos de $P$. c. patulus un metro por encima de la base de la Fm. Comabella, en la sección del barranco del Coll de Pal (Sanz, 1996).

\section{II) UNIDAD TECTÓNICA DEL COMTE-FREIXE (LES NOGUERES)}

Las primeras capas de calizas rosáceas sobre calizas amarillentas, en la parte inferior de la Fm. Villech de la sección de La Guardia, contienen Mimagoniatites sp. Este nivel se dispone por encima de la muestra 110 para conodontos de Boersma (1973), que probablemente se correlaciona con la Zona de $P$. gronbergi de Klapper (1977).

\section{III) UNIDAD TECTÓNICA DE ELS CASTELLS (LES NOGUERES)}

En la pista a Baén, al norte de la masía de Vilesa, se ha encontrado un ejemplar de Sellanarcestes cf. tenuior Walliser. Éste procede de niveles nodulares carbonatados entre margas verdes, localizados en la parte superior de la Fm. Villech. La parte alta de la Fm. Villech, en otras localidades cercanas, ha suministrado conodontos de la parte alta del Emsiense Superior. Esta edad es acorde con la que posee el taxón nominado en otras áreas geográficas (cf. Chlupác y Turek, 1983; Goddertz, 1987).

Por su parte, varios especímenes de Latanarcestes noeggerathi han sido recolectados en un tramo de tres metros de calizas nodulares y margas rojas de la parte superior de la Fm. Villech, en la sección de Els Castells. Los conodontos extraídos por Boersma (1973), en la 


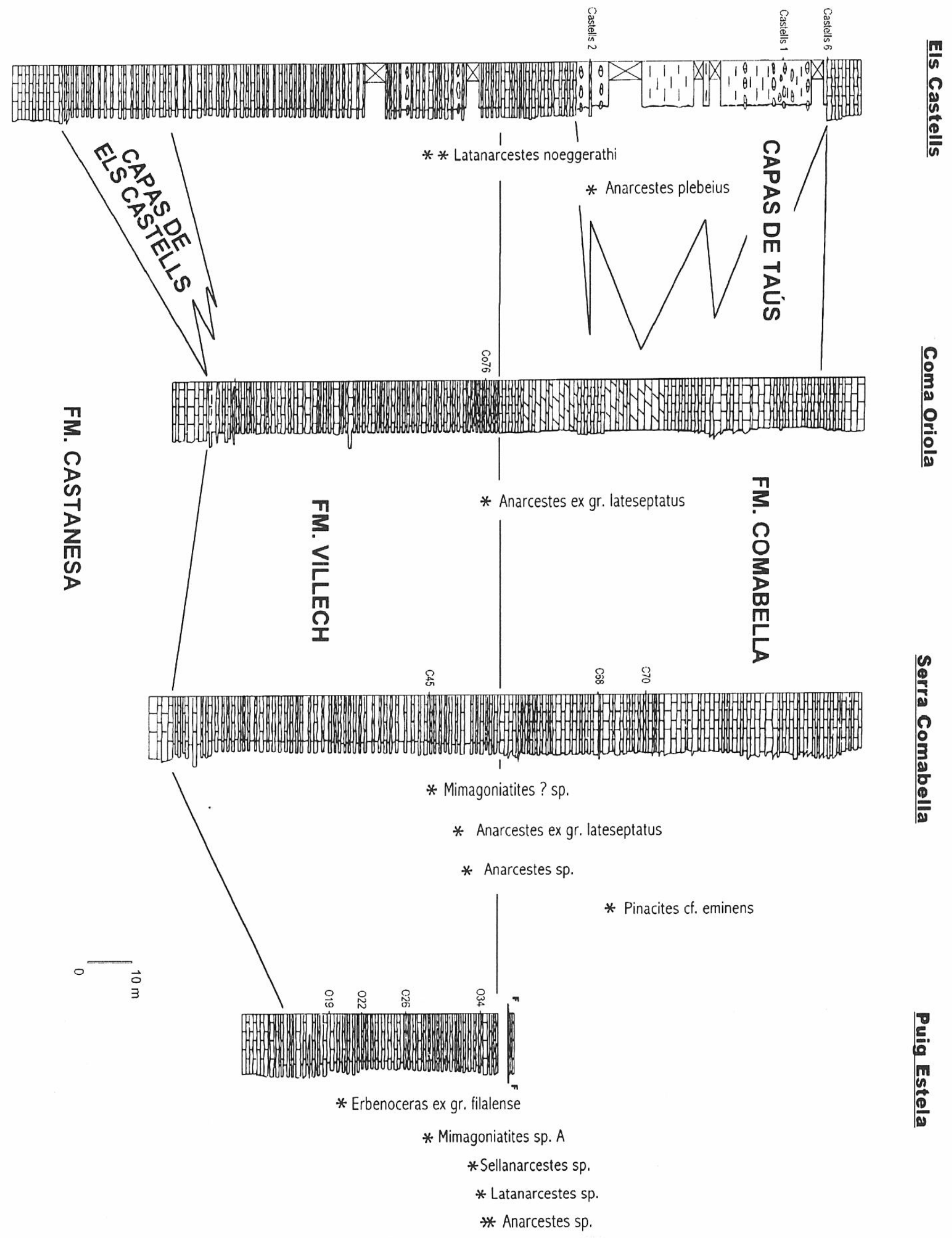

Figura 2. Columnas estratigráficas y distribución de la fauna de ammonoideos en varias sucesiones devónicas del Pirineo. La situación de las mismas se encuentra en la figura 1. 
parte alta de la Fm. Villech son atribuidos a la Zona de $P$. serotinus por Sanz (1996). Latanarcestes noeggerathi ha sido citada por autores previos en capas del Emsiense Superior. En la Península Ibérica Latanarcestes noeggerathi es conocida en capas del Miembro Lezna de la Fm. Abadía (Kullmann, 1960; Henn, 1985; Montesinos,1989), capas que Henn (1985) atribuyó a las biozonas de Nowakia richteri de dacrioconáridos y $P$. serotinus (probablemente inferior) de conodontos, ambas del Emsiense Superior.

Anarcestes plebeius (Barrande) está presente en una capa de caliza de $20 \mathrm{~cm}$ de espesor de la parte inferior de las Capas de Taús. A. plebeius se distribuye en secciones centroeuropeas y africanas entre la parte más alta del Emsiense Superior y la parte más baja del Eifeliense. Comparativamente, en la península Ibérica (Dominio Palentino, Cordillera Cantábrica), es conocido en capas de la Biozona de Polygnathus costatus partitus de Weddige (1977), en la parte basal del Eifeliense. En la sección estudiada yace en una capa atribuida indiferentemente a la Biozona de P. costatus (parte culminante del Emsiense Superior - Eifeliense "inferiormedio"), por la presencia de Polygnathus serotinus Telford $y$ un fragmento de $P$. costatus. Las margas que componen las Capas de Tahús extienden su registro hasta la Biozona de conodontos de $P$. ensensis, de manera similar a lo que sucede en el Dominio Palentino (Fm. Gustalapiedra) de la Zona Cantábrica (García-Alcalde et al., 1991), donde ha sido reconocido el Acontecimiento Pinacites (auct.) =jugleri de Walliser (1985).

\section{DESCRIPCIÓN DE LA FAUNA}

Los ammonoideos estudiados estarán depositados en la colección del Departamento de Geología de la Universidad de Oviedo. Por ello las referencias a los ejemplares comienzan mediante las letras DPO: Departamento de Paleontología de Oviedo. Las abreviaturas utilizadas son: $\mathrm{D}=$ diámetro de la concha; $\mathrm{E}=$ anchura de la vuelta; $\mathrm{H}=$ altura de la vuelta; $\mathrm{h}=$ altura interna de la vuelta; $\mathrm{O}=$ diámetro del ombligo. La edad de los taxones descritos ya ha sido discutida en el apartado anterior.

ORDEN ANARCESTIDA Miller y Furnish, 1954

SUBORDEN AGONIATITINA Ruzhentsev, 1957

Superfamilia Mimocerataceae Steinmann, 1890

Familia Mimosphinctidae Erben, 1953

Género Erbenoceras Bogoslovski, 1962

Erbenoceras ex gr. filalense

(G. Termier y H. Termier, 1950)

Lám I, fig. 2

Material: Un ejemplar conservado como molde externo calcáreo, con pequeñas porciones del molde interno, referencia DPO 113.907.

Yacimiento: Parte inferior de la Fm. Villech en la sección del Puig Estela. Capa situada tres metros por encima del nivel O19.
Descripción: Concha grande $(\mathrm{D}=68 \mathrm{~mm}$ aprox., $\mathrm{O}=45$ $\mathrm{mm}$ ), evoluta en los últimos estadios de crecimiento observados. En un diámetro inferior $(D=39 \mathrm{~mm})$, se aprecia que el enrollamiento de la concha es advoluto, por entrar en contacto la última vuelta con la precedente. La mala calidad de conservación del ejemplar impide la obtención de otras dimensiones y establecer sus relaciones paramétricas.

La ornamentación es muy densa, con costillas anchas y de fuerte relieve, que se inclinan hacia la parte posterior de la espira. La densidad de ornamentación es de aproximadamente 22 costillas en media vuelta a un diámetro de aproximadamente $41 \mathrm{~mm}$. Las costillas, en un diámetro de $68 \mathrm{~mm}$, tienen entre sí una separación de $0,5 \mathrm{~mm}$ y su anchura aproximada es de $3,2 \mathrm{~mm}$.

Discusión: El espécimen se encuentra muy mal conservado, pero le perduran caracteres suficientes, tanto en morfología de la concha como en la forma y regularidad de la ornamentación, para asignarlo al género Erbenoceras. Dentro de las especies de Erbenoceras, es relacionable con el que aquí se denomina "Grupo de Erbenoceras filalense", caracterizado por poseer elementos con un doble enrollamiento (evoluto/advoluto o sólo advoluto) en su ontogenia, que engloba especies tales como: E. filalense (Termier y Termier) [= Anetoceras advolvens (Erben)] y formas afines, tales como E. aff. advolvens Erben y Erbenoceras sp. A, B, C, D Erben, Erbenoceras solitarium (Barrande), Erbenoceras ellipticum Shen, Erbenoceras lineare Shen, Erbenoceras elegantulum Shen, Erbenoceras mattei Feist y Erbenoceras erbeni House.

Superfamilia Agoniatitaceae Holzapfel, 1899

Familia Mimagoniatitidae Miller, 1938

Género Mimagoniatites Eichenberg, 1930

\section{Mimagoniatites sp. A Lám. I, figs. 3 y 4}

Material: Un ejemplar, DPO 113.906, conservado como molde interno calcáreo.

Yacimiento: Parte media de la mitad superior de la Fm. Villech, en la sección del Puig Estela, Serra Caballera, 800 $\mathrm{m}$ al norte de la masía del Joncar. Pirineos orientales.

Descripción: Concha discoidal, fuertemente comprimida lateralmente. Margen ventral estrecho, redondeado. Flancos laterales muy amplios y suavemente convexos. Expansión de la espira, rápida. Ombligo moderado en anchura, con flancos umbilicales redondeados. Área de solapamiento mal observable, reducida.

De la sutura sólo es observable su porción externa: los lóbulos ventral y lateral. El lóbulo ventral es estrecho, en forma de V, de moderado desarrollo; el lóbulo lateral es de carácter omnilateral, redondeado y poco profundo.

El diámetro de la concha es de $54 \mathrm{~mm}$. Otras dimensiones son: $\mathrm{O}=13 \mathrm{~mm}, \mathrm{H}=29 \mathrm{~mm}$ y $\mathrm{E}=10 \mathrm{~mm}$ (aprox.). 
Lámina I
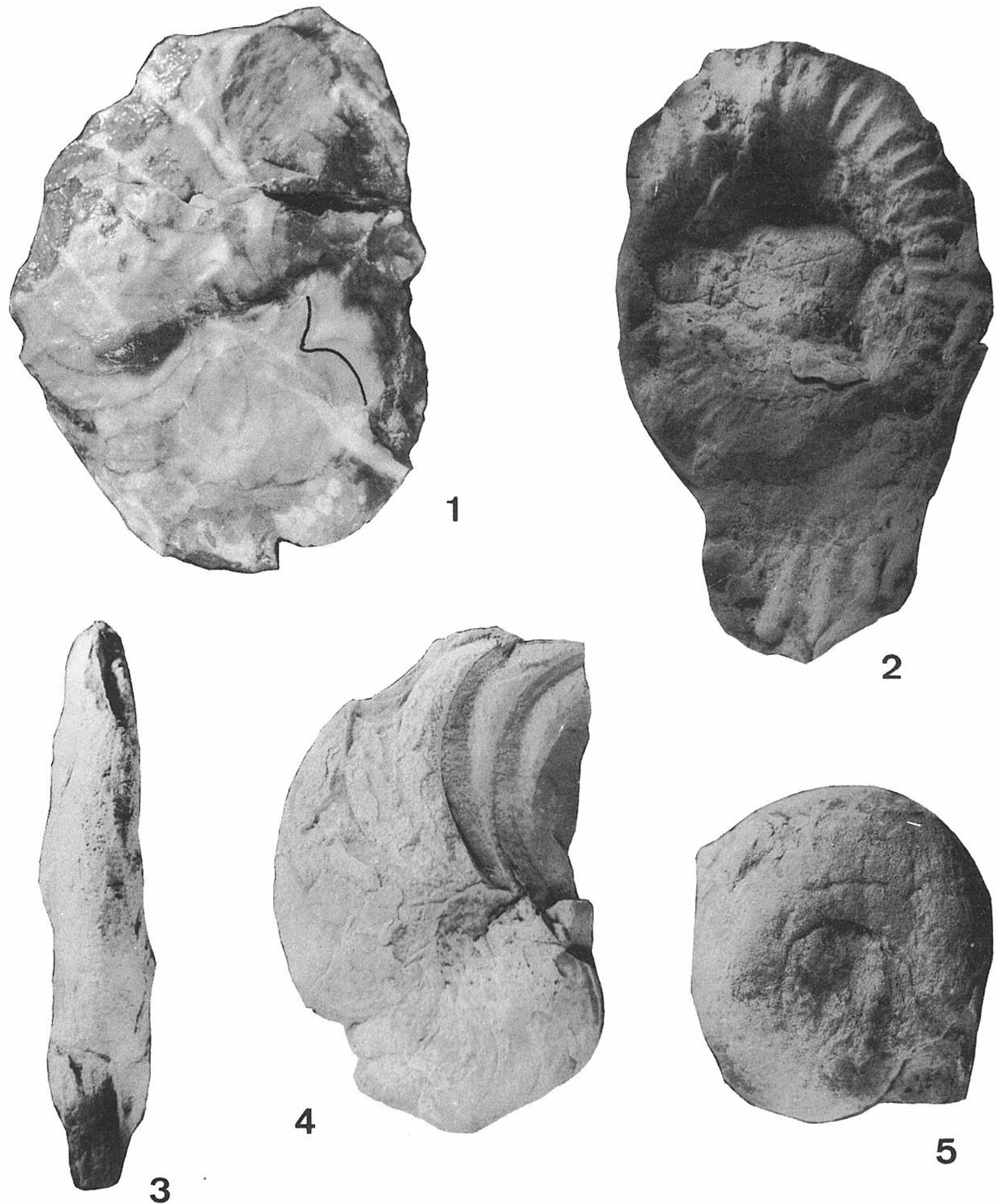

5

\section{Lámina I}

1 Pinacites cf. eminens Chlupác y Turek, 1977. Ejemplar DPO 113.905 de la parte inferior de la Fm. Comabella, fotografiado embebido en alcohol para resaltar las suturas. Sutura parcialmente retocada. Vista lateral derecha. $\mathrm{x} 1,5$.

2 Erbenoceras ex gr. filalense (G. Termier y H. Termier, 1950). Réplica en látex. Ejemplar DPO 113.907 en vista lateral izquierda. Parte inferior de la Fm. Villech. Ejemplar invertido para conservar la norma de iluminación. x1,5

3-4 Mimagoniatites sp. A. Ejemplar DPO 113.906 de la parte media de la Fm. Villech. 3. Vista ventral y 4. vista lateral derecha. x1,5.

5 Latanarcestes noeggerathi (Buch, 1832). Ejemplar DPO 113.911 de la parte superior de la Fm. Villech. Vista lateral derecha. x2.

Revista Española de Paleontología, nº extr. Homenaje al Prof. J. Truyols, 1999. 
Discusión: Mimagoniatites sp. A es una especie morfológicamente muy próxima a $M$. tabuliformis Kullmann, de la parte más alta del Emsiense Inferior, diferenciándose por poseer la primera una mayör tasa de expansión de las vueltas.

Un taxón también morfológicamente muy próximo, de similar edad, es el descrito por Montesinos (1989) como Mimagoniatites sp. A, del Dominio Palentino de la Cordillera Cantábrica, citado en el Miembro Lezna de la Formación Abadía. Este espécimen, muy fragmentario, muestra también una concha muy comprimida, sutura de similar trazado, expansión rápida y una sucinta área de solapamiento. En cualquier caso, el mal estado de conservación del material impide una comparación más precisa.

\section{SUBORDEN ANARCESTINA Miller y Furnish, 1954}

Superfamilia Anarcestacea Steinmann, 1890

Familia Anarcestidae Steinmann, 1890

Género Anarcestes Mojsisovics, 1882

\section{Anarcestes plebeius (Barrande, 1865)}

*1865 Goniatites plebeius Barr.; Barrande, lám. 5 figs. 9?, 16, 17, 18, 22; lám. 6, figs. 1-3.

1983 Anarcestes plebeius (Barrande); Chlupác y Turek, p. 91; lám. 24, figs. 1-10; lám 29, fig. 8; lám. 30, fig. 8; fig. text. 21. Designan lectotipo y lo figuran en lám. 24, fígs. 9-10; lám. 29, fig. 8 .

.1987 Anarcestes plebeius (Barrande); Göddertz, p. 157; lám. 6, fig. 5a-b; fig. text. 15 .

Lectotipo: Ejemplar figurado por Barrande (1865; lám 5, figs. 16-17), designado por Chlupác y Turek (1983).

Material: Tres ejemplares, DPO 113.902 a 113.904 , conservados como moldes internos carbonatados.

Yacimiento: Capa calcárea de $20 \mathrm{~cm}$ de espesor de la base de las Capas de Taús, en la sección de Els Castells, en las inmediaciones de la localidad del mismo nombre, $\mathrm{N}$ de la provincia de Lérida.

Descripción: Concha subdiscoide, túmida, con enrollamiento convoluto que presenta una zona de solapamiento bien desarrollada. La última vuelta del ejemplar DPO 113.904 es subreniforme, mientras que la previa es reniforme. La expansión de la espira es moderada.

Margen ventral redondeado, muy amplio, sin solución de continuidad con los flancos laterales. Flancos laterales cortos y muy convexos. Ombligo amplio. Bordes umbilicales redondeados. El flanco umbilical es rectilíneo y prácticamente perpendicular a los flancos laterales de la concha. Sutura no conservada en ninguno de los tres especímenes.

\section{Dimensiones y parámetros:}

$\begin{array}{lllllll}\text { DPO } & \text { D } & \text { E } & \text { H } & \text { O } & \text { O/D } & \text { E/H } \\ 113.902 & 50 & - & 18 & 20 & 0,4 & - \\ 113.903 & 52 & 22 & 16,5 & 21 & 0,4 & 1,33 \\ 113.904 & - & 14 & 11,5 & - & - & 1,21\end{array}$

Discusión: La forma general de la concha es común en algunos grupos de anarcéstidos, pero el tipo de expansión de la espira, el carácter prácticamente plano del flanco umbilical y su ángulo de incidencia con el flanco lateral, conjuntamente con las dimensiones de la concha, permiten vincular nuestro material con el género Anarcestes y, dentro de él, con la especie Anarcestes plebeius Barrande.

Género Latanarcestes Schindewolf, 1933

\section{Latanarcestes noeggerathi (Buch, 1832) Lám. I, fig. 5}

*1832 Ammonites Noeggerathi; Buch, p. 34; lám. 1, figs. 6-8.

Material: Cinco ejemplares fragmentarios, deformados, conservados como moldes internos calcáreos, DPO 113.909 a 113.913

Yacimiento: Parte superior de la Fm. Villech, en la localidad leridana de Els Castells.

Descripción: Concha discoidal. Expansión de las vueltas moderadamente rápida. Enrollamiento convoluto, con zona de solapamiento bien desarrollada, moderadamente profunda. Margen ventral redondeado. Flancos suavemente convexos. La sección de las vueltas es subtrapezoidal. Ombligo amplio con flancos umbilicales redondeados. Sutura mal conservada, con presencia en uno de los ejemplares de un lóbulo lateral redondeado, amplio y ligeramente polarizado hacia la región umbilical.

\section{Dimensiones (en mm):}

$\begin{array}{lllll}\text { DPO } & \text { D } & \text { E } & \text { H } & \text { O } \\ 113.911 & 27,2 & 12 & 11 & 9,8 \\ 113.909 & - & 6 & 5,5 & 6,4\end{array}$

Discusión: Las características generales de la concha, así como sus dimensiones, permiten identificar el material con el taxón nominado.

Género Sellanarcestes Schindewolf, 1933

\section{Sellanarcestes cf. tenuior Walliser, 1965}

Material: Un ejemplar DPO 113.908, conservado como molde interno calcáreo.

Yacimiento: Parte superior de la Fm. Villech, en capas nodulares carbonatadas entre margas verdes. Pista entre Bresca y Baén, a $100 \mathrm{~m}$ al $\mathrm{N}$ de la masía de Vilesa, $\mathrm{N}$ de la provincia de Lérida.

Descripción: Concha túmida, con margen ventral muy ancho y redondeado, sin solución de continuidad con los flancos laterales. Flancos laterales marcadamente convexos. Reborde umbilical carente de estructuras. Sección de la última vuelta subreniforme. Zona de solapamiento entre las vueltas moderadamente profunda. 
Sutura, observable con dificultad en el molde del probablemente último tabique, con un lóbulo lateral bien desarrollado, con aspecto redondeado y tendente a poseer una condición omnilateral.

\section{Dimensiones (aprox. en mm):}

$\begin{array}{lllll}\mathrm{D} & \mathrm{E} & \mathrm{H} & \mathrm{h} & \mathrm{O} \\ 45 & 17,5 & 1,35 & 1,05 & 21,5\end{array}$

Discusión: La forma de la concha, sus dimensiones y el trazado de la sutura lateral permiten relacionar el ejemplar con el género Sellanarcestes y, en concreto, con la especie $S$. tenuior Walliser. La pobre conservación del espécimen aconseja adoptar en su determinación un sistema de nomenclatura abierto.

Familia Pinacitidae Hyatt, 1900

Género Pinacites Mojsisovics, 1882

\section{Pinacites eminens Chlupác y Turek, 1977}

*1977 Pinacites eminens sp. n.; Chlupác y Turek, p. 304; lám. 1, figs. 1-4; fig.-text. 1 .

Discusión: Chlupác y Turek (1977) establecieron como carácter diagnóstico exclusivo del taxón, y diferencial con respecto a especies cogenéricas, la presencia en $P$. eminens de lóbulos accesorios en los flancos del lóbulo ventral. Otro carácter que probablemente puede ser diagnóstico de la especie es la forma del lóbulo umbilical, que en el caso de $P$. eminens tiene el flanco lateroumbilical de la silla $\mathrm{L} / \mathrm{U}$ con una fuerte pendiente, que hace que el aspecto del lóbulo umbilical sea más profundo que aquel que posee $P$. jugleri (Roemer); este último taxón posee una pendiente más suave en la rama L/U, observable en las ilustraciones del taxón auct. y en el material propio de investigación, y que origina un lóbulo umbilical de aspecto más somero (ver Roemer, 1843 y Chlupác y Turek, 1977, 1983).

La carencia de descripciones y figuras del taxón nominado en la bibliografía reciente no aconseja, por el momento, realizar una diagnosis enmendada a aquella que ofrecieron Chlupác y Turek (1977).

\section{Pinacites cf. eminens Chlupác y Turek, 1977 Lám I, fig. 1}

Material: Un molde interno calcáreo con DPO 113.905, en mal estado de conservación.

Yacimiento: Muestra situada cuatro metros por encima del yacimiento C68 de la parte inferior de la Fm. Comabella, en la vertiente meridional de la Sierra de Comabella, $100 \mathrm{~m}$ al sur del Collado Creu d'en Galofré, Pirineo oriental.

Descripción: Concha oxicónica, lateralmente comprimida, de flancos amplios, ligeramente convexos. Ombligo estrecho, mal conservado. La sutura lateral es bilobada, con un lóbulo lateral amplio, redondeado y moderadamente profundo. Silla latero-umbilical subaguda con una rama umbilical de fuerte pendiente. Lóbulo umbilical amplio, redondeado y profundo. Lóbulo ventral no conservado.

Diámetro de la concha $58 \mathrm{~mm}$ (aprox.) y anchura mínima de $9 \mathrm{~mm}$.

Discusión: La forma de la concha y el trazado de la sutura lateral permiten incluir nuestro ejemplar en el género Pinacites. Existen tres especies asignadas al género nominado: $P$. jugleri, $P$. eminens y $P$. irideum (Frech). Nuestro ejemplar se diferencia de $P$. irideum en la forma de la silla L/U que, tal como indicaron Chlupác y Turek (1983, p. 99), tiene el aspecto propio de géneros tales como Foordites Wedekind, 1918, Wedekindella Schindewolf, 1928, y otros relacionados, con una silla E/L marcadamente asimétrica, con una rama umbilical muy horizontal. De $P$. jugleri se diferencia en el aspecto del lóbulo umbilical y de la silla E/L, en el sentido que se indica más arriba en la discusión del taxón nominal.

La conservación extremadamente pobre del ejemplar descrito y el que no se pueda observar la forma del lóbulo ventral, hace que la determinación se realice en nomenclatura abierta.

\section{CONCLUSIONES}

Del estudio de las distintas secciones citadas en el texto se desprende que la fauna de ammonoideos del Devónico Inferior/Medio en el Pirineo catalán es relativamente abundante y con una diversidad equiparable a otros afloramientos devónicos de la península Ibérica. De todas formas, pese a esa abundancia, la calidad de la fauna es bastante pobre, pues los ejemplares se encuentran normalmente deformados y destruidos más o menos parcialmente por causa de fenómenos de metamorfismo regional. Excepcionalmente existen secciones en las cuales el grado de conservación es relativamente bueno y que necesitarían de un tipo de estudio mucho más exhaustivo que el que se ha planteado para el presente trabajo.

El conjunto de los especímenes citados en el texto ha sido recolectado en sedimentos de tipo hemipelágico de la Fm. Villech y parte inferior de la Fm. Comabella. La mayor abundancia local de fauna, tanto en número de ejemplares como en diversidad, se produce en las calizas nodulares hemipelágicas de la parte superior de la Formación Villech, que pertenecen a la Biozona de $P$. serotinus de conodontos. Diferentes niveles de este conjunto han proporcionado ejemplares de Agoniatites, Anarcestes, Mimagoniatites y Sellanarcestes. El último género es citado por primera vez en el ámbito del Pirineo. Este conjunto de fauna corresponde al horizonte de Anarcestes subnautilinus en el sentido de Dalloni (1913, 1930), que en realidad integra varios tramos con ammonoideos, localizados en la parte superior de la Formación Villech, donde se ha obtenido Latanarcestes noeggerathi, y en la parte inferior de la Fm. Comabella del Pirineo central y oriental (Emsiense Superior). 
Otros ammonoideos, como los ejemplares de Erbenoceras y Pinacites, tienen una localización puntual y están relacionados con facies de calizas nodulares. La presencia de Erbenoceras es particularmente interesante para el conocimiento de la distribución de faunas primitivas de ammonoideos en el Devónico Inferior de la península Ibérica, mientras que, por su parte, Pinacites es un género guía de la base del Devónico Medio (Eifeliense). La edad de las asociaciones de ammonoideos estudiadas es totalmente equiparable con lo conocido previamente en otros yacimientos ibéricos y de otras partes del mundo (Centroeuropa, África del Norte, por ej.).

\section{AGRADECIMIENTOS}

Agradacemos a los Dres. Jürgen Kullmann y Jacques Thierry, sus valiosas sugerencias y revisión del escrito original. Así mismo, agradecemos al Nationaal Natuurhistorisch Museum de Leiden y en especial al Dr. Marinus van den Boogaard, las facilidades y apoyo recibido durante las observaciones sobre conodontos de la colección de K.Th. Boersma. Este trabajo es una contribución a los proyectos de la Dirección General de Enseñanza Superior del Ministerio de Educación DGE-PB94-1324 y DGE-PB951047 y al Proyecto P.I.C.G. núm. 421 "North Gondwana Mid-Palaeozoic Bioevent/Biogeography patterns in relation to crustal dynamics".

\section{ANEXO DE LAS UNIDADES LITOESTRATIGRÁFICAS ( J. Sanz)}

Formación Villech.- La Fm. Villech fue definida por Hartelvelt (1970) en el Tosal de Víllec (al sur del río Segre, unidad del Segre), para capas de calizas rojas, lutitas y calizas nodulares. Esta unidad se encontraba sobre las calizas grises de la Fm. Basibé (aquí Fm. Castanesa, ambas de Mey, 1967) y el Mb. A de la Fm. Compte. La potencia de la formación variaba entre 80 y 125 metros y fue atribuida a un intervalo entre el Emsiense y el Givetiense. Más tarde, la sección (o más bien área) tipo fue estudiada por Boersma (1973), que argumentó que estaba mal seleccionada, ya que la sucesión está muy distorsionada y semicubierta. Este autor realizó algunas secciones entre Castellar de N'Hug y la Noguera Pallaresa, donde obtuvo conodontos que atribuyó al intervalo entre el Emsiense Inferior y Eifeliense superior. Sanz (1996) encontró en el área del Pirineo oriental meridional, una potencia menor para la Fm. Villech que la afirmada por Hartelvelt (1970). Dicho espesor varía entre 30-40 m en las secciones entre Castellar de N'Hug y Camprodon, a $75 \mathrm{~m}$ hacia la Tosa d'Alp. La base de la Fm. Villech se sitúa en los primeros tramos margosos, de espesor decimétrico, que suceden a la Fm. Castanesa, mientras que el criterio utilizado por Boersma (1973), coloración roja, se adquiere unos metros por encima. De esta manera, el tramo inferior está compuesto por 4-8 $\mathrm{m}$ de calizas nodulares de tentaculítidos que pueden intercalar margas grises o amarillas y es sucedido en la vertical por calizas marrones más arcillosas y calizas rosas nodulares "lime mud- wackestone". Encima, se dispone la litología típica de la formación, alternancias decimétricas a centimétricas de margas rojas y calizas amarillentas o rosas. La fauna está dominada por dacrioconáridos con escasos fragmentos de trilobites, crinoides, briozoos, ostrácodos, gasterópodos, algún ortocerátido, escasos corales rugosos solitarios pequeños de forma cerioide y corales tabulados coloniales masivos. En la parte superior de la formación, los ciclos decimétricos de alternancias de calizas y margas son estratocrecientes, con una predominancia del componente calcáreo. Las calizas y margas contienen horizontes con cefalópodos enrollados de hasta 25 centímetros de diámetro. Los niveles más pelíticos son de color marrón claro, verde o amarillo. En el área de Castellar de N'Hug, como en otras secciones orientales, la parte más alta de la sucesión está formada por calizas moradas bioturbadas, que contienen una gran cantidad de tentaculítidos, cefalópodos y algunos moldes de bivalvos grandes, que llegan a ser más abundantes en la parte inferior de la formación suprayacente.

Formación Comabella.- La Formación Calizas de Comabella es definida en la vertiente meridional de la Serra de Comabella, Collada de la Bofia y Morriador de Cabanes (al suroeste de la Tosa d'Alp). Otras secciones de la formación se encuentran descritas en Sanz (1996). Esta formación equivale aproximadamente al Miembro A de la Fm. Compte de Hartelvelt (1970), definida en los alrededores de la masía de El Comte (Gerri, valle de la Noguera Pallaresa). El límite con la Fm. Villech es transicional y frecuentemente ocupa una posición más baja en las sucesiones que el utilizado por Boersma (1973) para la base de la Fm. Compte. La Fm. Comabella está compuesta por calizas nodulares versicolores, entre las que dominan las calizas de tonos grises claros. Las capas de lutitas o margas son escasas y con potencias del orden de un centímetro como máximo. En Castellar de N'Hug, la base de la Fm. Comabella se ha situado en una caliza gris clara o beige, sobre la que se disponen dos metros de calizas grisesmoradas, nodulares, en bancos de 3-6 centímetros con gran cantidad de tentaculítidos ("wackestone-packstone"), cefalópodos de 6-8 centímetros de diámetro, dispuestos horizontalmente, algunos moldes de bivalvos de gran tamaño y bioturbación. Al oeste de Castellar y hasta Béixec las calizas nodulares presentan frecuentes intercalaciones de calizas crinoidales o calizas coralinas. Estos tramos predominan en la parte media y superior de la formación. Las potencias así mismo varían entre 20 metros, cuando está representada exclusivamente por calizas nodulares, a cerca de 370 metros, en las secciones entre Gréixer y Penyes Altes del Moixeró. En todas las sucesiones, los metros finales están representados por calizas nodulares, que en la sección de Comabella soportan un "grainstone" bioclástico de cuarenta centímetros, con braquiópodos y cefalópodos. La base de esta capa es el límite de la formación y sobre ella, se disponen calizas nodulares rojas con cefalópodos. La edad de la formación abarca del Emsiense Superior al Frasniense superior o Fameniense basal.

Capas de Castells.- Unidad formada por lutitas y margas grises azules u ocres, que intercalan capas centimétricas a 
decimétricas de calizas arcillosas ocres ("wacke-packstone" de dacrioconáridos). Su potencia en la sección de Els Castells, donde ha sido estudiada, es de 30 metros. Se dispone sobre la Fm. Castanesa, por un aumento en las capas de lutitas, que llegan a ser de escala decimétrica. Su parte más alta es más carbonatada, con calizas rosadas, verdes o marrones, entre margas grises claras con tonos rosáceos, que anteceden a la Fm. Villech. Contienen faunas de conodontos, dacrioconáridos, ostrácodos, trilobites, pequeños corales solitarios y están muy bioturbadas. Las especies de conodontos citadas en Boersma (1973) señalan una edad Emsiense Inferior.

Capas de Taús.- Unidad formada por lutitas grises azuladas a negras y margas grises verdosas o de coloraciones ocres por meteorización, con una potencia de 40 metros. Se dispone sobre 17 metros en Els Castells, de calizas nodulares grises claras, correlacionadas con la parte inferior de la Fm. Comabella. La base de la formación está marcada por lutitas en capas de más de 5 centímetros y en esta parte inferior se intercalan algunas capas de calizas nodulares. Las lutitas pueden contener horizontes de nódulos o capas de calizas arcillosas, en mayor número hacia su parte alta. Su techo se coloca en la base de calizas nodulares con dacrioconáridos de la Fm. Comabella. Esta unidad contiene faunas de dacrioconáridos, cefalópodos, conodontos y trilobites, abundantes en su parte superior. La edad de estas capas es Eifeliense. Su base podría situarse todavía en el Emsiense Superior (Zona de $P$. c . patulus) ya que no se dispone de datos precisos. Su techo se encuentra por debajo de una asociación de la Zona de $P$. ensensis. Las muestras de Boersma (1973) 254 y 255, revisadas en el Museo de Leiden, contienen, a nuestro parecer, Icriodus werneri Weddige e I. struvei Weddige.

\section{BIBLIOGRAFÍA}

Barrande, J. 1865-1867. Système silurien du centre de la Bohême, II, Classe des Mollusques, ordre des Cephalopodes. Lám. 1-107 (1865), pp. 1-712 (1867). Praga, París.

Boersma, K.Th. 1973. Devonian and Lower Carboniferous conodont biostratigraphy, Spanish Central Pyrenees. Leidse Geologische Mededelingen, 49, 303-377.

Bouquet, C. et Stoppel, D. 1975. Contribution à l'étude du Paléozoïque des Pyrénées centrales (Hautes vallées de la Garonne et d'Aure). Bulletin du Bureau de Recherches Géologiques et Minières de la France, 2 (1), 7-61.

Buch, L. von, 1832. Über Goniatiten. Abhandlungen der Akademie der Wissenschaften, Berlín, 159-187.

Bultynck, P. 1989. Conodonts from the La Grange Limestone (Emsian), Armorican Massif, North-Western France. Courier Forschungsinstitut Senckenberg, 117, 173-203.

Casas, J.M., Domingo, F., Poblet, J. and Soler, A. 1989. On the role of the Hercynian and Alpine thrusts in the Upper Paleozoic rocks of the Central and Eastern Pyrenees. Geodinámica Acta (Paris), 3(2), 135-147.

Chlupác, I., 1976. The oldest goniatite faunas and their stratigraphical significance. Lethaia, 9, 303-315.

Chlupác, I. and Turek, V. 1977. New cephalopods (Ammonoidea, Bactritoidea) from the Devonian of the Barrandian area, Czechoslovakia. Vestnik Ústredniho ústavu geologického, 52, 303-306.

Chlupác, I. and Turek, V. 1983. Devonian goniatites from the Barrandian area Czechoslovakia. Ústredniho ústavu geologického, 46, 1-159.

Dalloni, M. 1910. Etude géologique des Pyrénées de l'Aragon. Annales de la Faculté des Sciences de Marseille, 19, 1-444.

Dalloni, M. 1911. Sur l'extension des griottes á Anarcestes subnautilinus dans les Pyrénées. Bulletin de la Societé géologique de la France, 11, p. 68.

Dalloni, M. 1913. Stratigraphie et tectonique de la région des Nogueras (Pyrénées centrales). Bulletin de la Societé géologique de la France, Paris, 4(13), 243-263.

Dalloni, M. 1930. Etude géologique des Pyrénées catalanes. Annales de la Faculté des Sciences de Marseille, 26 (3), $1-373$.

García-Alcalde, J., Montesinos, J.R., Truyols-Massoni, M., García-López, S., Arbizu, M.A. and Soto, F. 1991. The Palentine Domain (Palentian Zone). In: Pre-mesozoic Geology of Iberia (Eds. R.D. Dallmeyer and E. Martínez García), Springer Verlag, Berlín, 20-23.

Göddertz, B. 1987. Devonischen Goniatiten aus SWAlgerien und ihre stratigraphische einordnung in die Conodonten-Abfolge, Palaeontographica, Abt. A, 197 (4-6), 127-220.

Hartevelt, J.J.A. 1970. Geology of the Upper Segre and Valira valleys, Central Pyrenees, Andorra/Spain. Leidse Geologische Mededelingen, 45, 167-236.

Henn, A.H. 1985. Biostratigraphie und Fazies des höhen Unter-Devon bis tiefen Ober-Devon der Provinz Palencia, Kantabrisches Gebirge, N-Spanien. Göttinger Arbeiten zur Geologie und Paläontologie, 26, 1-100.

Klapper, G. 1977. Lower and Middle Devonian conodont sequence in Central Nevada, with contributions by D.B. Johnson. In: Western North America: Devonian (Eds. M.A. Murphy, W.B.N. Berry and C.A. Sandberg). University of California Riverside Campus Museum Contributions, 4, 33-54.

Klapper, G., Ziegler, W. and Mashkova, T.V. 1978. Conodonts and correlation of Lower-Middle Devonian boundary beds in Barrandian area of Czechoslovakia. Geologica et Palaeontologica, 12, 103-116.

Kullmann, J. 1960. Die Ammonoidea des Devons in Kantabrischen Gebirge (Nord-spanien). Abhandlungen der Mathematisch-Naturwissenschaftlichen Klasse, 7, 1106.

Kullmann, J. und Calzada, S. 1982. Goniatiten (Cephal.) aus herzynischem Unter-devon der Ost-Pyrenäen. Neues Jahrbuch für Geologie und Paläontologie Monatshefte, 1982, 593-599.

Mey, P.H.W. 1967. The Geology of the Upper Ribagorzana and Baliera valleys, Central Pyrenees, Spain. Leidse Geologische Mededelingen, Leiden, 41, 153-220.

Mey, P.H.W. 1968. Geology of the Upper Ribagorzana and Tor valleys, Central Pyrenees, Spain. Leidse Geologische Mededelingen, 4, 229-292. 
Montesinos, J.R. 1989. Las Biozonas de ammonoideos del Devónico (Emsiense Inferior-Fameniense Inferior): crítica al sistema de clasificación zonal. Revista Española de Paleontología, 5, 3-17.

Muñoz, J.A. 1985. Estructura alpina i herciniana a la vora sud de la zona axial del Pirineu oriental. Tesis Doctoral, Univ. de Barcelona, 1-305 (inédita).

Muñoz, J.A. 1992. Evolution of a Continental Collision Belt: ECORS-Pyrenees Crustal Balanced Cross-section. In: Thrusts Tectonics (Ed. K.R. Mc Clay). Champan \& Hall, 235-246.

Roemer, F.A. 1843. Die Versteinerungen des Harzgebirges. I-XX, 1-40. Hannover.

Sanz, J. 1996. Estratigrafía (Conodontos) del Silúrico Superior - Carbonífero Inferior del Pirineo Oriental y Central. Tesis Doctorales microfichadas núm. 2840. Publicaciones de la Universitat de Barcelona, 9 p., 3 microfichas. Tesis Doctoral 1995, $701 \mathrm{pp}$.

Schmidt, H. 1931. Das Paläozoikum der spanischen Pyrenäen. Abhandlungen aus der Gesellschaft der Wissenschaften zu Göttingen. MathematischPhysikalische Klasse, 3 (5), 981-1065. Publicaciones alemanas sobre Geología de España (1944), 101-202.

Seguret, M. 1972. Étude tectonique des nappes et séries décollées de la partie centrale du versant sud des
Pyrénées. Caractère synsédimentarie, rôle de la compression et de la gravité. Publ USTELA, Série Géol. struct., Montpelier, 2 1-155.

Termier, G. et Termier, H. 1950. Invertebrés de l'Ère Primaire. Paléontologie Marocaine, T. II, Fasc. III, Mollusques. Actualités Scientifiques et Industrielles, 1094, 1-246.

Valero, J. 1974. Géologie structurale du Paléozoïque de la région de Panticosa. Province de Huesca (Espagne). Thèse 3ème cycle, Bordeaux, 1-78 (inédita).

Walliser, O.H. 1965. Uber Sellanarcestes Schindewolf 1933 (Ammonoidea, Unter-bis Mitteldevon). Fortschritte in der Geologie von Rheinland und Westfalen Krefeld, 9 , 87-96.

Walliser, O.H. 1985. Natural boundaries and Commission boundaries in the Devonian. Courier Forschungsinstitut Senckenberg, 75, 401-408.

Weddige, K. 1977. Die Conodonten der Eifel-Stufe im Typusgebiet und in benachbarten Faziesgebieten. Seckenbergiana lethaea, 58 (4/5), 271-419.

Wensink, H. 1962. Paleozoic of the Gallego and Ara Valleys, Huesca province, Spanish Pyrenees. Estudios Geológicos, Madrid, 18 (1-2), 1-74.

Zwart, H.J. 1979. The Geology of the Central Pyrenees. Leidse Geologische Mededelingen, 50, 1-74. 